\begin{tabular}{|c|c|}
\hline 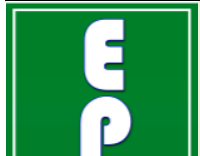 & $\begin{array}{c}\text { International Journal of Current Research } \\
\text { and Academic Review }\end{array}$ \\
\hline $\begin{array}{l}\text { EXCELLENT } \\
\text { PUBLLSHERS }\end{array}$ & $\begin{array}{c}\text { ISSN: 2347-3215 (Online) Volume } 7 \text { Number } 4 \text { (April-2019) } \\
\text { Journal homepage: } \text { http://www.ijcrar.com }\end{array}$ \\
\hline
\end{tabular}

doi: https://doi.org/10.20546/ijcrar.2019.704.002

\title{
A Review on Bio Prospective Potential of Corynebacterium glutamicum
}

\author{
Asfaw Tora Kacho ${ }^{1}$ and B.S.R. Pattnaik ${ }^{2 *}$ \\ ${ }^{1}$ Applied Microbiology, Department of Biology, College of Natural and Computational Science, Wolaita Sodo \\ University, Ethiopia \\ ${ }^{2}$ Department of Biology, Wolaita Sodo University, Ethiopia \\ *Corresponding author
}

\section{Abstract}

Corynebacterium glutamicumis a nonpathogenic, aerobic, gram-positive soil bacterium used for the large-scale biotechnological production of several biomolecules. C. glutamicum is an industrial microbe traditionally used for the production of amino acids. It is a well known microbe as the workhorse in fermentation industry used since its first discovery in Japan in 1950s for production of monosodium glutamate (MSG)and L-lysine with a market size of 3.1 and 2.2 million. However, for the fermentative production of diverse products through geneticmetabolic engineering. Due to its industrial importance, several clones of $C$. glutamicum have been sequenced by both industry and academic groups. Furthermore it has also been exploited for the synthesis of a variety of other fuels and chemicals. Especially, the facultative anaerobic lifestyle of this Gram-positive bacterium formed the basis to engineer C. glutamicum for the production of reduced molecules such as organic acids (e.g., lactate, succinate) and alcohols (e.g., ethanol, isobutanol) under zero-growth anaerobic conditions. The present work is a review of various uses of $C$. glutamicum for which the researchers explored several resources including the PubMed database, Journals and online archives upto March 2019. The review in its analysis signifies the vital utility of $C$. glutamicum in various prospective. Most articles were on occurrence, metabolism, metabolic role, and industrial uses of bacterial polyhydroxyalkanoates. Through genetic alterations, this microbe showed its efficacy for the simultaneous utilization of hexose and pentose sugars of biomass hydrolysate for making value-added products, such as amino acids and polyamines, and thus making it a strain of choice to work in a bio-refinery concept. This soil microbe also showed its efficacy in bioremediation purposes, such as arsenic removal. Recently its extensive research has focused on engineering beyond the scope of amino acids for utilization of alternative carbon sources, (e.g. coming from wastes and unprocessed sources), and construction of $C$. glutamicum strains for production of new products such as diamines, organic acids and alcohols. Meanwhile, a variety of corynebacterial strains allows access to alternative carbon sources that allows production of a wide range of industrially relevant compounds. Some of these efforts set new standards in terms of titers and productivities achieved whereas others represent a proof-of-principle. These achievements manifest the position of $C$. glutamicum as an important industrial microorganism with capabilities far beyond the traditional amino acid production. According to the studies cited in different literatures $C$. glutamicum reported as the most effective and playing important roles as dominant bacteria in biotechnological applications as well as industrial processes and products. And, with recent advances in biotechnology, the economic contributions that these organisms can make in biotechnological applications and industrial processes can be exploited further for large scale benefit of mankind.
\end{abstract}

\section{Article Info}

Accepted: 04 March 2019

Available Online: 20 April 2019

\section{Keywords}

Aerobiosis, Bioprospecting, Corynebacterium glutamicum, Microaerobiosis, Metabolic engineering, Triple-phase process. 


\section{Introduction}

Corynebacterium glutamicum is an industrial microbe traditionally used for the production of amino acids. It proved its potential, however, for the fermentative production of diverse products through geneticmetabolic engineering. Due to their snapping type of division, cells often lie in clusters resembling chinese letters. Corynebacteria are chemoorganotrophic, aerobic, or facultatively anaerobic, and they exhibit a fermentative metabolism (carbohydrates to lactic acid) under certain conditions. They are fastidious organisms, growing slowly on even an enriched medium.

It was first discovered as a producer of glutamate. Now it is used to make amino acids, such as lysine, threonine, and isoleucine, as well as vitamins like pantothenate. Through genetic alterations, this microbe showed its efficacy for the simultaneous utilization of hexose and pentose sugars of biomass hydrolysate for making valueadded products, such as amino acids and polyamines, and thus making it a strain of choice to work in a biorefinery concept. This soil microbe also showed its efficacy in bioremediation purposes, such as arsenic removal.

Corynebacterium glutamicum is used industrially for large-scale production of amino acids (Akashi et al., 1978). While originally identified in a screen for organisms secreting L-glutamate, mutants of $C$. glutamicum have also been identified which produce various other amino acids. Hydroxybenzoic acids are industrially relevant aromatic compounds, which also play key roles in the microbial carbon metabolism, e.g., as precursors for the synthesis of cofactors or metalchelating molecules. Due to its pronounced resistance to aromatics Corynebacterium glutamicumrepresents an interesting platform for production of these compounds. Unfortunately, a complex catabolic network for aromatic molecules prevents application of $C$. glutamicumfor microbial production of aromatic compounds other than aromatic amino acids, which cannot be metabolized by this microorganism.

\section{Taxonomy}

C. glutamicum belong to Kingdom Bacterium, Phylum Actinobacteria, Order Actinomycetales, Sub-order Corynebacteriaceae, Genus Corynebacterium, Species glutamicum. Corynebacteria are small, generally nonmotile, Gram-positive, rod-shaped (ellipsoidal) bacteria non-sporulating (although they have club-like ends), pleomorphic bacilli and is aerobic in nature.

\section{Abundance}

Many species of Corynebacteria can be isolated basically from places such as soil, water, blood, and human skin. C. glutamicum strains isolated from soil contaminated with bird feces and manure. Pathogenic strains of Corynebacteria can infect plants, animals or humans. However, human are now the only known reservoir for the disease. The bacterium is generally found in temperate zones but may also be found in other parts of the world. Nondiptherial Corynebacteria are ubiquitous in nature, and are commonly found in human mucous membranes and skin. Due to its industrial importance several clones of $C$. glutamicum have been sequenced by both industry and academic groups(Anderson AJ, Dawes EA., 1990)

\section{Industrial Production of Amino Acids Using Corynebacterium glutamicum}

1-Amino acids find various applications in biotechnology. 1-Glutamic acid and its salts are used as flavor enhancers. Other l-amino acids are used as food or feed additives, in parenteral nutrition or as building blocks for the chemical and pharmaceutical industries. 1amino acids are synthesized from precursors of central carbon metabolism. Based on the knowledge of the biochemical pathways microbial fermentation processes of food, feed and pharma amino acids have been developed. The organism Corynebacterium glutamicum is used in industry to produce L-lysine through an aerobic process (Ahmed et al., 2012). The biosynthesis of L-Lysine, in which a pathway is followed leading from aspartate (which is itself derived from oxaloacetate, a Krebs cycle intermediate) through a series of intermediates, all the way to L-Lysine. Pertinent enzymes are shown on the left, and this shows that significant attention can be expended on strain development and metabolic pathway engineering to tune the expression of all of these components and boost production.

According to Anastassiadis (2007), "Process improvement for producing larger amounts of L-lysine using microorganisms remains a continual attempt, whereas the continuous development of classical and modern genetics resulted in the development of superior strains imparted with properties advantageous for the 
commercial production of L-lysine." For this project a culture will be ordered from a cell culture bank, such as the American Type Culture Collection. An example culture that is known to be effective is labeled as ATCC13032. The organism selected to carry out the production of L-lysine is $C$. glutamicum. The strains of this bacteria typically form pale yellow colonies, however some cream-white strands can also occur.

This organism is widely used in the production of Llysine because it is able to metabolize a variety of carbohydrates, alcohols and organic acids as carbon and energy sources for growth and for amino acid production. It is also able to metabolize inorganic nitrogen sources such as ammonia. L-lysine is produced in about seven or ten steps from its precursor oxalacetate. C. glutamicum has a respiratory metabolism, with oxygen as its terminal electron acceptor. Production strains of Corynebacterium glutamicum, which has been used safely for more than 50 years in food biotechnology, and Escherichia coli are constantly improved using metabolic engineering approaches. Fermentative production of 1-amino acids in the millionton-scale has shaped modern biotechnology and its markets continue to grow steadily (Akinterinwa et al., 2008).

\section{Bio-refinery Applications of Corynebacterium glutamicum}

The bio-refinery concept is an emerging concept for conducting industrial processes to manufacture a range of commodity chemicals, fuels, and energy from biomass-based feedstock. The current interest in implementing a biorefinery industry is largely derived by a combination of rising petroleum prices as well as the need to reduce greenhouse gas emissions and atmospheric $\mathrm{CO}_{2}$ levels to mitigate global warming. To date, Corynebacterium glutamicum-based technology has not been considered as the primary manufacturing platform for sustainable chemicals. Indeed, despite a long history of use for the industrial production of amino acids, C. glutamicum, as compared to Escherichia coli or Saccharomyces cerevisiae, has been scarcely studied and engineered to fit the needs of the lingo-cellulosic biorefinery. However, progress over the last decade in the understanding of its molecular physiology and metabolic engineering makes this microorganism an attractive option as a biorefinery biocatalyst. In addition, the development of a novel bioprocess using growth-arrested cells of $C$. glutamicum under oxygen deprivation constitutes a promise for bio-refinery research and development (Alper Hand Stephanopoulos, 2009).

\section{Recent trend}

Metabolic Engineering of Corynebacterium glutamicumnow used as a designer-bug for the bio-based production of chemical building blocks and biofuel (Alper Hand Stephanopoulos G, 2009).Corynebacterium glutamicumis used also for the production of hydroxybenzoic acids (Kallscheuer and Marienhagen). Hydroxybenzoic acids are industrially relevant aromatic compounds, which also play key roles in themicrobial carbon metabolism, e.g., as precursors for the synthesis of cofactors or metal-chelating molecules. Due toits pronounced resistance to aromatics $C$. glutamicum represents an interesting platform for production of these compounds (Kallscheuer et al., 2018) also finished the construction of the platform strain $C$. glutamicumDelAro5,in which the deletion of altogether 27 genes in five gene clusters abolished most of the peripheral and central catabolic pathways for aromatic compounds known in this microorganism. The obtained strain was subsequently applied for the production of2hydroxybenzoate (salicylate), 3-hydroxybenzoate, 4hydroxybenzoate and protocatechuate, which all derive from intermediates of the aromatic amino acid-forming shikimate pathway. For an optimal connection of the designed hydroxybenzoate production pathways to the host metabolism, C. glutamicum was additionally engineered towards increased supply of the shikimate pathway substrates erythrose-4-phosphate and phosphoenolpyruvate by manipulation of the glucose transport and key enzymatic activities of the central carbon metabolism.

By abolishing its natural catabolic network for aromatic compounds, $C$. glutamic was turned into a versatile microbial platform for aromatics production, which could be exemplarily demonstrated by rapidly engineering this platform organism towards producing four biotechnologically interesting hydroxybenzoates. Production of these compounds was optimized following different metabolic engineering strategies leading to increased precursor availability. The constructed $C$. glutamicumstrains are promising hosts for the production of hydroxybenzoates and other aromatic compounds at larger scales (Nicolai Kallscheuer and Jan Marienhagen, 2018).

Deciphering the Adaptation of Corynebacterium glutamicum in Transition from Aerobiosis via 
Microaerobiosis to Anaerobiosis was done by Julian Lange et.al. They investigated the adaptation of Corynebacterium glutamicum to altering oxygen availabilities, we conceived a triple-phase fermentation process that describes a gradual reduction of dissolved oxygen with a shift from aerobiosis via microaerobiosis to anaerobiosis. During the process, sequential samples were drawn at six points and analyzed via RNAsequencing, for metabolite concentrations and for enzyme activities. We found transcriptional alterations of almost 50\% (1421 genes) of the entire protein coding genes and observed an upregulation of fermentative pathways, a rearrangement of respiration, and mitigation of the basic cellular mechanisms such as transcription, translation and replication as a transient response related to the installed oxygen dependent process phases (Julia Wennerhold and Michael Bott, 2018).

The DtxRRegulon of Corynebacterium glutamicum was established by Julia (Wennerhold et al., 2006). Comparative analysis of the C.glutamicum group and complete genome sequence of strain $\mathrm{R}$ was done by (Hideaki et al., 2007). The complete genome sequence of Corynebacterium glutamicum strain $\mathrm{R}$ was determined to allowits comparative analysis with other corynebacteria. The discovery of 39 novel genes in C. glutamicum R that have not been previously reported in other corynebacteria supports the rationale for sequencing additional corynebacterial genomes to better define the corynebacterial pan-genome and identify previously undetected metabolic pathways in these organisms.

The dawn of a new era in corynebacterial research in which genome wide genetic manipulations by Manipulating Corynebacteria from Individual Genes to Chromosomes forming a complete genome sequence that have the potential to generate more efficient and more versatile whole-cell industrial biocatalysts become possible. To strengthen the biotechnological attributes of C.glutamicum, whose potential to serve as a biocatalyst of commodity chemicals has recently been demonstrated, the versatility of the corynebacterial transcription/translation system offers the possibility to adapt tools developed in E. coli and B. subtilis.

However, the further improvement of corynebacteriummediated bioconversions requires a greater understanding of corynebacterial physiology, such as catabolite repression or the fine details of the global regulation of gene expression and protein activity (Ahmed Zahoor et al., 2012) described the Metabolic engineering of Corynebacterium glutamicum aimed at alternative carbon sources and new products such as alcohols, proteins and biofuels etc.

It is evident that currently there is plentiful research going on to metabolically engineer $C$. glutamicum production of more than two-third of the products discussed in this review has been made possible in the last few years. The availability of the genome sequence and genetic engineering tools for this organism make its rapid and rational manipulation possible. So far, 'omics' technologies have not been extensively used in context of metabolic engineering of this bacterium, whereas they do hold the potential to elucidate production mechanisms and constraints on a systems-wide level.

\section{Conclusion}

According to the studies cited in different literatures $C$. glutamicum reported as the most effective and playing important roles as dominant bacteria in biotechnological applications as well as industrial processes and products. And, with recent advances in biotechnology, the economic contributions that these organisms can make in biotechnological applications and industrial processes can be exploited further for large scale benefit of mankind. Therefore, we encourage any bio prospecting company to access this genetic resource for its potentials in biotechnological applications as well as industrial processes and products for access and benefit sharing. The described transcriptional adaptation of C.glutamicum resolved to varying oxygen availabilities provides a useful basis for future process and strain engineering. Glutamic acid was traditionally extracted from seaweed to serve as a seasoning agent and remains to this day a very important food flavor additive, the worldwide production of which approximates 1.5 million tons per year (Alain, 2005).

The utilization of recombinant DNA techniques, combined with metabolic and carbon flux analyses, facilitated the identification of metabolic bottlenecks and their bypassing by expressing or repressing the corresponding genes to develop further improved amino acid industrial production processes.

Studies on the regulation of C.glutamicum genes and enzymes involved in the citric acid cycle, which is of central importance for metabolism in general and for amino acid production in particular because it provides the precursors of the aspartate and glutamate family of amino acids (Julia Wennerhold and Michael Bott., 2018). 


\section{References}

1. Ahmed Zahoor a, Steffen N. Lindner a, Volker F. Wendisch; Metabolic engineering of Corynebacterium glutamicum aimed at alternative carbon sources and new products, Computational and Structural Biotechnology Journal | www.csbj.org, Volume No: 3, Issue: 4, October 2012.

2. .Alain A. Verte`s, Masayuki Inui, and Hideaki Yukawa; Manipulating Corynebacteria, from Individual Genes to Chromosomes, applied and environmental microbiology, Dec. 2005, p. 7633-7642 Vol. 71, No. 12,2005.

3. Akashi K, Ikeda S, Shibai H, Kobayashi K, Hirose Y (1978) Determination of redox potential levels critical for cell respiration and suitable for L-leucine production. Biotechnol Bioeng 20:27-41.

4. Akinterinwa O, Khankal R, Cirino PC (2008) Metabolic engineering for bioproduction of sugar alcohols. Curr Opin Biotechnol 19:461467.

5. Alper H, Stephanopoulos G (2009) Engineering for biofuels: exploiting innate microbial capacity or importing biosynthetic potential? Nat Rev Microbiol 7:715-723

6. Anderson AJ, Dawes EA (1990) Occurrence, metabolism, metabolic role, and industrial uses of bacterial polyhydroxyalkanoates. Microbiol Rev 54:450-472

7. Anastassiadis, S. L-Lysine Fermentation. 2007. Recent Patents on Biotechnology 1: 11-24.

\section{How to cite this article:}

Asfaw Tora Kacho and Pattnaik, B.S.R. 2019. A Review on Bio Prospective Potential of Corynebacterium glutamicum. Int.J.Curr.Res.Aca.Rev. 7(4), 7-11.

doi: https://doi.org/10.20546/ijcrar.2019.704.002
8. Aristidou A, Penttilä M (2000) Metabolic engineering applications to renewable resource utilization. Curr. Opin Biotechnol 11:187-198

9. Hideaki Yukawa, Crispinus A. Omumasaba, Hiroshi Nonaka; Comparative analysis of the C.glutamicum group and complete genome sequence of strain R, Microbiology (2007), 153, 1042-1058 DOI 10.1099

10. Julian Lange, Eugenia Münch, Jan Müller, Tobias Busche, JörnKalinowski, Ralf Takors and Bastian Blombach,; Deciphering the Adaptation of Corynebacterium glutamicum in Transition from Aerobiosis via Microaerobiosis to Anaerobiosis, Genes 2018, 9, 297; doi: 10.339

11. Julia Wennerhold and Michael Bott; The DtxR Regulon of Corynebacterium glutamicumjournal of bacteriology, Apr. 2006, p. 2907-2918 Vol. 188, No-8, 0021-9193, 2006

12. Käß, F., S. Junne, P. Neubauer, W. Wiechert, and M. Oldiges. 2014. Process inhomogeneity leads to rapid side product turnover in cultivation of Corynebacterium glutamicum. Microbial Cell Factories. 13:6.

13. Kjeldsen. 2008. Optimization of an industrial Llysine producing Corynebacterium glutamicum strain. Technical University of Denmark, Department of Systems Biology.

14. Nicolai Kallscheuer and Jan Marienhagen, Corynebacterium glutamicumas platform for the production of hydroxybenzoic acids, Microb Cell Fact (2018) 